\title{
Steroid Resistant Takayasu Arteritis
}

\author{
Dasgupta $M K^{1}$, Das $S^{2}$, Guha $D^{3}$ \\ ${ }^{1}$ Dr. Malay Kumar Dasgupta, MBBS, DCH, MD, \\ Professor, ${ }^{2}$ Dr. Sabyasachi Das, MBBS, MD, Senior \\ Resident, ${ }^{3} \mathrm{Dr}$. Debasree Guha, MBBS, MD, RMO. All \\ from the Department of Paediatrics. R.G. Kar Medical \\ College and Hospital, 1, Khudiram Bose Sarani. \\ Kolkata-04, India.
}

\section{Introduction}

$\mathrm{T}$ akayasu's arteritis (TA), is a large vessel vasculitis, more common in Orient, mainly Asia'. It has been reported in adults of every age specially in adolescent. More than half of patients $(60 \%)$ with Takayasu arteritis achieve control on corticosteroids alone, some proportion of cases are Glucocorticoid resistant ${ }^{2}$. This disease is the commonest cause of renovascular hypertension in Asian children ${ }^{2,3}$. Here we report such a case requiring a steroid sparing immunosuppressive for disease control.

\section{The Case}

Our case is a 12 year old girl admitted with a history of sudden onset weakness of left side of the body for 2 days with inability to sit, associated with headache and vomiting. On asking there was also a history of pain in different joints and irregular fever for 8-10 months. Other history was uneventful.

General examination revealed wasting and stunting ( Wt- $19 \mathrm{~kg}$, Height - $128 \mathrm{~cm}$, BMl- $12 \mathrm{~kg} / \mathrm{m}^{2}$-all are $<5^{\text {th }}$ percentile for age), pallor and absence of all pulses of upper limb (Radial, Brachial), bilaterally weak femoral pulses, pulsation of right carotid artery is totally absent, in left side it is palpable with a thrill and a bruit on auscultation. Blood pressure could not be measured clinically. On neurological examination positive findings were left sided UMN type of facial nerve palsy, UMN type of weakness of left upper and lower limb (Power $3 / 5$ ), without any sensory loss. Weakness was improved over few days and she was able to walk within a week. Based on these clinical features we diagnosed the case as Vasculitc Syndrome (Primary/Secondary) with CNS vascular insufficiency.

\begin{abstract}
Takayasu's arteritis (TA), a chronic inflammatory disease affecting the aorta, its branches and the pulmonary arteries has become increasingly recognized as a worldwide entity, with a variable spectrum of disease expression. Here in a case of paediatric Takayasu arteritis affecting purely the arch of aorta and it's branches that was steroid resistant.
\end{abstract}

Investigation showed- $\mathrm{Hb} \%-9.65 \mathrm{gm} \%$, normal WBC \& platelet count, high ESR-102/hr, positive CRP (> $52.4 \mathrm{mg} / \mathrm{dl}$ ), negative ASO, RA factor, anti-phospholipid antibody, ANCA ( $p \& c$ ). Serum biochemistry including sugar, urea, creatinine, LFT, RFT was normal so as chest X-ray, ECG, echocardiography. CT scan revealed ischemic changes in right basal ganglion region. Angiography was done to delineate the vessel involvement. It showed narrowing of subclavian arteries bilaterally with extensive collateral formation, right carotid artery was totally non-visualised and in left side it was severely narrowed. Tracing below abdominal aorta showed normal diameter of vessels blood pressure measured during this procedure was high 140/90 mm of $\mathrm{Hg}$.

As soon as diagnosis was confirmed, treatment was started with oral prednisolone $2 \mathrm{mg} / \mathrm{kg}$ and angiographic stenting of left carotid artery was contemplated but due to high risk for procedure consent was not given by parents, so only steroid was continued and after one month dose was gradually tapered but clinical response was poor. Review after 3 months of tapering dose, pulses were still not palpable, there were frequent history of headache, acute phase reactant was still high, and signs of steroid toxicity (Cushinoid facies, excessive weight gain) also was obvious. Pulse course of cyclophosphamide was started as steroid sparing agent, patient became symptom free within three months, acute phase reactant became negative but peripheral pulses didn't return till date (around a year).

\section{Address for correspondence \\ Dr. Sabyasachi Das \\ E-mail: dasbabai83@gmail.com}

\section{How to cite this article?}

Dasgupta MK, Das S, Guha D. Steroid Resistant Takayasu

Arteritis. J Nepal Paediatr Soc 2013;33(2):144-146. 


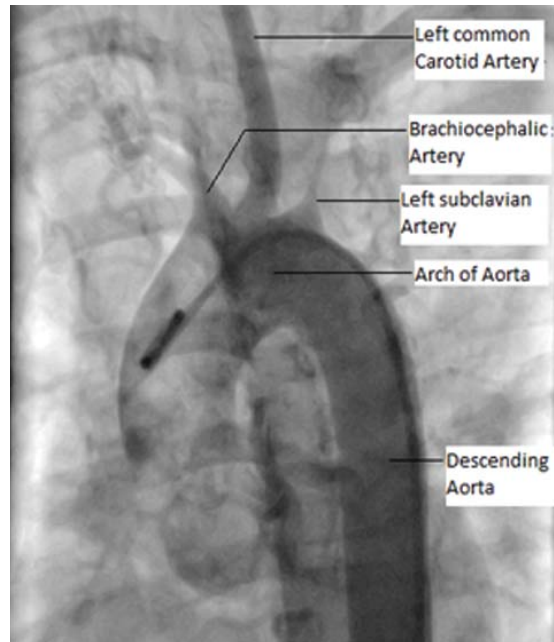

Fig 1: Branches of Arch of Aorta. Markedly narrowed Brachiocephalic artery and left Subclavian artery

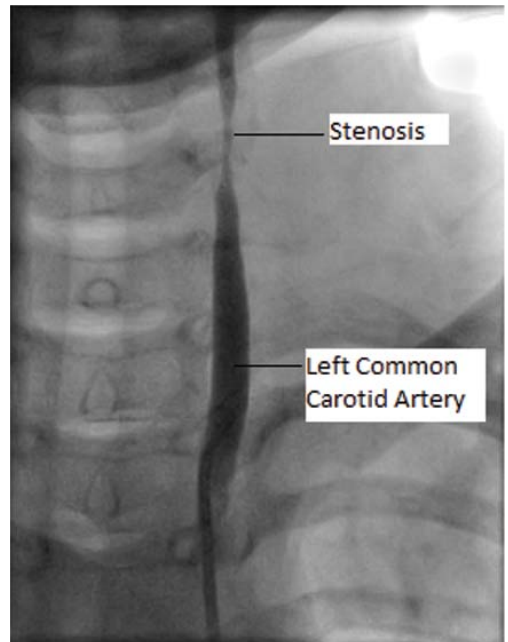

Fig 2: Marked narrowing of Left common carotid artery

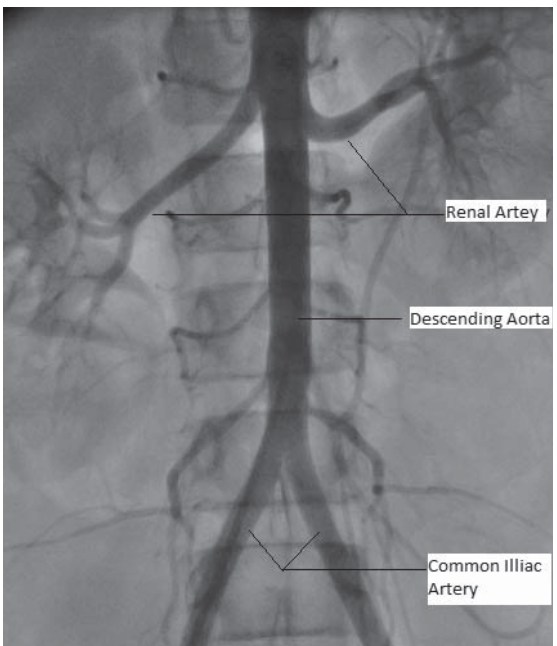

Fig 3: Normal descending Aorta and its branches
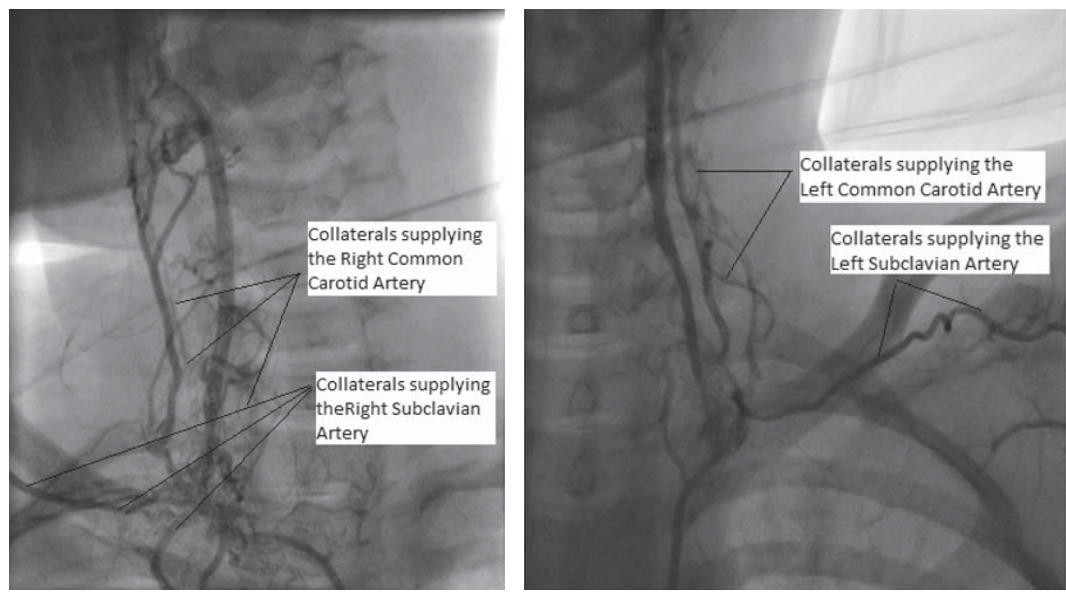

Fig 4: Extensive collateral circulations around the right and left Subclavian and Common Carotid Arteries.

\section{Discussion}

Takayasu arteritis affects the aorta and its major branches. The clinical presentation of TA includes three following phases-Pre-pulseless phase, pulseless phase and occlusive phase ${ }^{1}$. Exact etiopathogenesis of the disease is unknown ${ }^{2}$. Despite the term pulseless disease, which is a synonym for Takayasu arteritis, the predominant finding in individuals with Takayasu arteritis is asymmetrical pulse. Absent peripheral pulses occur late in the course of the disease.

Based on angiographic findings Takayasu's arteritis is divided into type I (involving aortic arch and its branches), type II (thoracoabdominal aorta and its branches) and type III (involving lesions of both type I \& II). Involvement of pulmonary arteries in addition to any of the above types is grouped as type IV. Thoracoabdominal aortic involvement is commoner (type II/III) in children². The unique character of our case is pure involvement of Aortic arch which is uncommon in the paediatric age group.

Takayasu's arteritis is the commonest cause of renovascular hypertension in Asian children ${ }^{2,3}$. Though in our case there was no renal artery involvement, this probably resulted from less renal blood flow due to narrowing at arch of aorta.

Angiogram is the gold standard for assistance in the diagnosis of Takayasu arteritis. It is performed either with conventional invasive angiography or CT angiography. Although the luminal changes of Takayasu arteritis are well depicted with conventional angiography, its mural changes can be best evaluated with spiral computed tomography (CT) angiography ${ }^{4}$. More recently MRI has been used to establish the diagnosis of TA in children, to monitor disease activity and to guide treatment ${ }^{5}$. 
High dose Glucocorticoid is mainstay of therapy for induction of remission as well as maintenance in low doses for 1 to 2 years ${ }^{1}$. Corticosteroid therapy can achieve clinical remission in up to $60 \%$ of patients ${ }^{2,6}$. However few patients not responding to corticosteroids or who relapse during corticosteroid taper or progressively develop steroid toxicity require non-steroid immunosuppressive drugs. If prednisolone cannot be tapered to an alternate day regimen after three months or if there is disease progression, drugs like cyclophosphamide $(2 \mathrm{mg} / \mathrm{kg} /$ day), azathioprin (100 mg/day) or low dose methotrexate $(0.3 \mathrm{mg} / \mathrm{kg} /$ week) may enhance the efficacy of steroid therapy ${ }^{7}$. Glucocorticoid resistance could be explained by the increased cytokine production ${ }^{8}$. Anti-TNF therapy may be a possible beneficial agent for these patients. Criteria for Glucocorticoid-resistance or Glucocorticoiddependence and for disease remission have not been uniformly defined. There is $s$ need for prospective randomised trials with larger patient groups, longer follow-up and well defined criteria for response and relapse, in order to be able to give evidence-based recommendations for patients not responding to Glucocorticoid alone in the future.

\section{Conclusion}

Takayasu arteritis is a type of large vessel vasculitis, common in Asian countries and present with characteristic asymmetrical or absent pulses. Most of the cases are steroid sensitive. The unique character of our case was pure involvement of only aortic arch and it branches, which is uncommon in paediatric age group.

\section{References}

1. Daniela Bartos, Ecaterina Bontas, S Ghiorghe, Cercetari Experimentale \& Medico-Chirurgicale 2006; Nr 3-4:149-152.

2. SS Kothari, Takayasu's arteritis in children-a review. Images Paediatr Cardiol 2001;3(4):4-23.

3. Chugh KS, Sakhuja V. Takayasu's arteritis as a cause of renovascular hypertension in Asian countries. Am J Nephrol 1992; 306: 464-65.

4. Chung JW, Kim HC, Choi YH, Kim SJ, L ee W, Park $\mathrm{JH}$. Patterns of aortic involvement in Takayasu arteritis and its clinical implications: evaluation with spiral computed tomography angiography. J Vasc Surg 2007; 45(5): 906-14.

5. Safia Al abrawi, Marine Fouillet-Desjonqueres, Louis David et al. Takayasu arteritis in children. Pediatric Rheumatol 2008,6:17.

6. Juergen Brunner, Brian M Feldman, Pascal N Tyrrell et al. Takayasu arteritis in children and adolescents. Rheumatology 2010;49(10):1806814.

7. Manotosh Panja, PC Monda. Current Status of Aortoarteritis in India, $J$ Assoc Phy India 2004(52);48-52

8. I Drigo, A Saccari, C Bacchin, E Barbi, et al. Glucocorticoid resistance in a girl with Takayasu's arteritis:_Ann Rheum Dis 2006;65(5):689-90. 